to SLEDAI-2K. SLE-DAS is quickly scored with its online calculator. An increase in SLE-DAS $\geq 1.72$ was validated as a clinically meaningful worsening of SLE disease activity. [2]

Objectives: To compare the performance of SLE-DAS, classic SFI (c-SFI), revised SFI (r-SFI) and SLEDAI-2K in the identification of lupus flares in a reallife clinical setting.

Methods: We included patients with SLE fulfilling classification criteria [ACR (1997) and/or SLICC and/or EULAR/ACR], followed at an academic lupus clinic from January 2017 to June 2020 , and presenting with lupus low disease activity score (LLDAS) at baseline.

Flares occurring after baseline were identified as fulfillment of the conceptual definition of flare, as assessed by the senior lupus expert at time of each outpatient visit. For each flare event, we evaluated the fulfillment of flare criteria according to c-SFI, r-SFI, SLEDAI-2K (score increase $\geq 4$ points from baseline), and SLE-DAS (score increase $\geq 1.72$ from baseline). As control visits without flare, we considered the first visit after baseline, where we assessed the four tools, excluding those where a flare was identified by the gold-standard expert evaluation. Sensitivity and specificity of the four flare tools were estimated and McNemar's test applied to assess differences with the gold-standard flare definition The inter-instrument agreement with the gold-standard was assessed through Cohen's Kappa.

Results: We included 297 patients (female: $86.2 \%$; mean age: $48.9 \pm 14.6$ years, mean disease duration: $12.5 \pm 9.0$ years). At baseline, all patients were in LLDAS receiving ongoing antimalarials, immunosuppressants, and/or glucocorticoids in $91.0 \%, 43.8 \%$ and $33.6 \%$, respectively. During follow-up, $22.2 \%$ developed flares. The analysis included 92 flares [musculoskeletal $(40.2 \%)$; renal $(23.9 \%)$ mucocutaneous $(18.5 \%)$, haematological $(5.9 \%)$, serositis $(3.3 \%)$; multisystemic $(8.7 \%)$ ], with increase or change of treatment in $80.4 \%$ of these episodes, and 292 visits without flare considered as control.

There was no statistically significant difference between either SLE-DAS flare or $c-S F I$ and the gold-standard expert flare definition $(p=0.41$ and $p=0.82$, respectively), while $\mathrm{r}$-SFI and SLEDAI-2K flare were different from the gold-standard (Table 1). There was a strong agreement between SLE-DAS flare, c-SFI, r-SFI and the expert definition (Cohen's kappa, Table 1).

Table 1. Performance of the flare tools for the gold-standard flare definition.

\begin{tabular}{lcccc}
\hline & Sensitivity (\%) & Specificity (\%) & McNemar's $^{\S \S}$ & Kappa $^{\S}$ \\
\hline SLE-DAS flare & 90.1 & 95.0 & n.s. & 0.836 \\
c-SFI flare & 91.1 & 96.5 & n.s. & 0.869 \\
r-SFI flare & 93.4 & 92.9 & $p<0.01$ & 0.820 \\
SLEDAI-2K flare & 51.6 & 98.6 & $p<0.0001$ & 0.590 \\
\hline
\end{tabular}

$\$ \S$ McNemar's test: significant difference from gold-standard if $p<0.05 ;{ }^{\S}$ Cohen's kappa agreement $(0-1)$ with the gold-standard flare definition; n.s.: non-significant

Conclusion: The c-SFI and SLE-DAS showed the best performance in identifying SLE flares. The SLE-DAS flare definition is easier to apply and hence might be considered as an optimal tool to be used in daily clinical practice.

REFERENCES:

[1] Ruperto N, Hanrahan LM, Alarcón GS, et al. International consensus for a definition of disease flare in lupus. Lupus. 2011;20(5):453-62.

[2] Jesus D, Matos A, Henriques C, et al. Derivation and validation of the SLE Disease Activity Score (SLE-DAS): a new SLE continuous measure with high sensitivity for changes in disease activity. Ann Rheum Dis 2019; 78:365-71.

Disclosure of Interests: None declared.

DOI: 10.1136/annrheumdis-2021-eular.748

OP0294

SJÖGREN'S SYNDROME ASSOCIATED LYMPHOMAS: CLINICAL DESCRIPTION AND 10-YEAR SURVIVAL

L. Chatzis ${ }^{1}$, V. Pezoulas ${ }^{2}$, A. Goules ${ }^{1}$, I. Stergiou ${ }^{1}$, C. Mavragani ${ }^{3}$,

G. Tsourouflis ${ }^{4}$, D. Fotiadis ${ }^{2}$, H. M. Moutsopoulos ${ }^{5}$, M. Voulgarelis ${ }^{1}$, A. Tzioufas ${ }^{1}$

${ }^{1}$ School of Medicine, National and Kapodistrian University of Athens,

Pathophysiology, Athens, Greece: ${ }^{2}$ University of loannina, Unit of Medical

Technology and Intelligent Information Systems, Athens, Greece; ${ }^{3}$ School of

Medicine, National and Kapodistrian University of Athens, Physiology, Athens,

Greece; ${ }^{4}$ School of Medicine, National and Kapodistrian University of Athens,

Second Department of Propaedeutic Surgery, Athens, Greece: ${ }^{5}$ Academy

of Athens, Chair Medical Sciences/Immunology, Chair Medical Sciences/ Immunology, Greece, Athens, Greece

Background: Sjögren's Syndrome (SS) is a chronic systemic autoimmune disease of unknown etiology, carrying the highest lymphoma risk among autoimmune diseases, with significant impact on mortality and morbidity of patients. Objectives: To describe: i) the clinical phenotype of SS, ii) the histologic type, stage, treatment options regarding lymphomas and iii) the prognosis of patients with SS related lymphoproliferative disorders.
Methods: Eight hundred and fifteen consecutive SS patients' records from a single center fulfilling the 2016 ACR/EULAR were reviewed retrospectively for the purpose of this study. One hundred twenty-one patients with a diagnosis of non-Hodgkin Lymphoma (NHL) were identified and enrolled in the study population. Cumulative clinical, laboratory and histologic data were recorded and overall survival as well as event free survival curves were constructed using the Kaplan-Meier method. An event was defined as a disease progression, lymphoma relapse, treatment failure, histologic transformation, development of a 2nd lymphoma or death from any cause.

Results: From 121 pSS patients with lymphoma the most common histologic type encountered was MALT lymphoma (92/121, 76,0\%) followed by DLBCL $(11 / 121,9.0 \%)$ and NMZL $(8 / 119,6.6 \%)$. The remaining 10 patients had various lymphomas of B (follicular, lymphoplasmacytic, chronic lymphocytic leukemia) and $\mathrm{T}$ cell origin (peripheral $\mathrm{T}$ cell lymphoma not otherwise specified, primary cutaneous $T$ cell lymphoma, angioimmunoblastic t-cell lymphoma). Permanent salivary gland enlargement $(66.1 \%, 80 / 121)$, palpable purpura $(34,7 \% 42 / 121)$ peripheral nervous involvement $(9,9 \%, 12 / 121)$, interstitial lung disease $(8,2 \%$, $10 / 121)$ presence of serum cryoglobulins $(38,7 \%, 43 / 111)$ and C4 hypocomplementemia $(69,8 \% 81 / 116)$ present at least 1 year before the development of lymphoma were the main pSS related features. The median age at lymphoma diagnosis was 58 years old (range 29-82) while MALT Iymphomas developed earlier compared to DLBCL from pSS diagnosis ( 8 vs $3 \mathrm{OR}=3.84,95 \% \mathrm{Cl}: 0.29$ to $10.46 ; p=0.0266)$. The commonest biopsy proven extranodal sites included the labial minor salivary $(43,8 \%$ patients) and parotid glands $(30,5 \%)$ while $11 \%$ of patients had more than 1 extranodal sites affected. Bone marrow involvemen was evident in $24,3 \%$ of patients (29/119) while nodal involvement in $35,5 \%$ (42/118). The majority of patients $(65 \%)$ had limited disease (stage I or II). A watch and wait therapeutic policy was chosen in 40 patients while the rest received rituximab with or without chemotherapy. The 10-year survival and even free rates were $79 \%$ and $45,5 \%$ for MALT lymphomas, $40,9 \%$ and $24,2 \%$ for DLBCL and $46 \%$ and $31 \%$ for NMZL respectively (Figure 1). The Mantel-Cox logrank comparison of the overall survival curves revealed a statistically significant difference $(p=0.0016)$ among lymphoma subtypes.

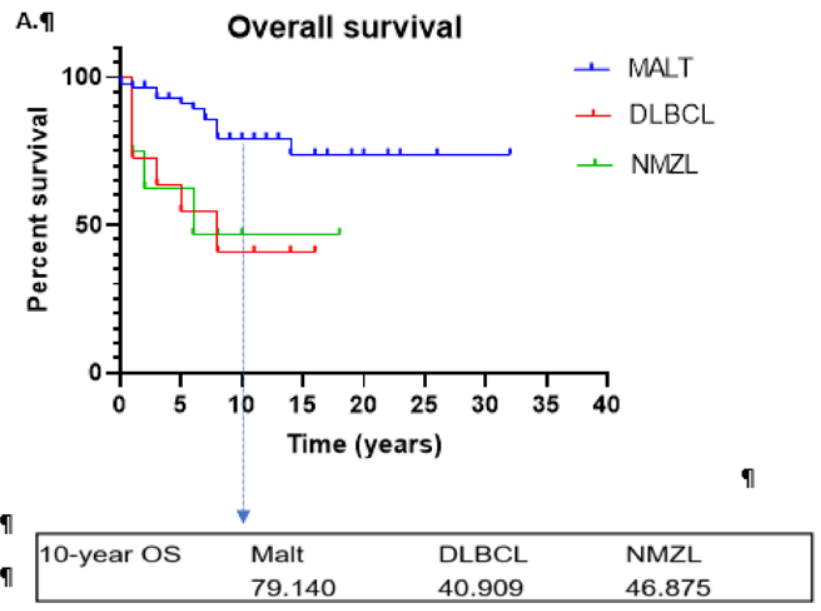

B.ๆ Event free survival

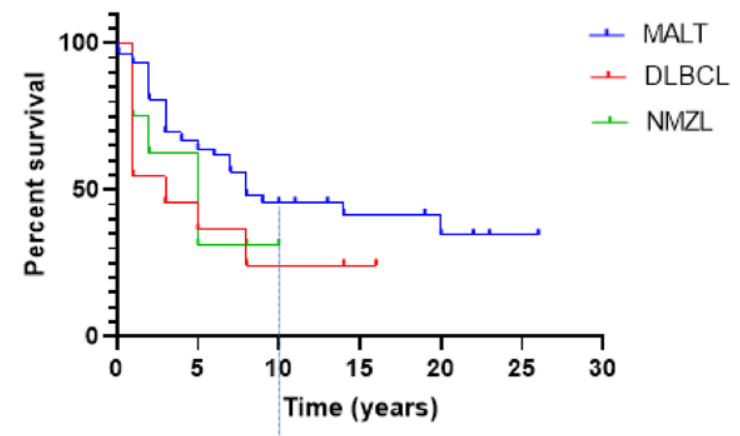

m

ๆ

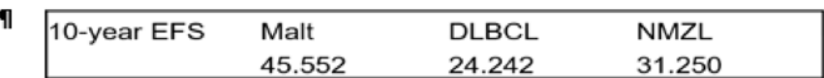

Figure 1. Overall and event free survival of SS-associated lymphoma patients. A. KaplanMeier overall survival analysis. B. A Kaplan-Meier event free survival analysis. 
Conclusion: This is the largest single center series of SS- associated lymphoma patients, providing a detailed description of SS and lymphoma related features, combined with a 10-year survival and event free curves for the first time in the literature.

Disclosure of Interests: None declared

DOI: 10.1136/annrheumdis-2021-eular.2400

\section{OP0295 GR2 MULTICENTRIC PROSPECTIVE FRENCH STUDY'S RESULTS: DAMAGE BUT NOT REMISSION AT FIRST TRIMESTER PREDICTS ADVERSE PREGNANCY OUTCOME IN LUPUS PREGNANCIES}

M. Larosa ${ }^{1,2}$, V. Le Guern ${ }^{1}$, G. Guettrot Imbert ${ }^{1}$, E. Lazaro ${ }^{3}$, N. Morel $^{1}$, N. Abisror Jeannin ${ }^{4}$, C. Morati-Hafsaoui ${ }^{5}$, P. Orquevaux ${ }^{6}$, E. Diot ${ }^{7}$, F. SarrotReynauld $^{8}$, A. Doria ${ }^{2}$, A. Moltó ${ }^{9}$, C. Deneux-Tharaux ${ }^{10}$, N. CostedoatChalumeau ${ }^{1}$ on behalf of GR2 Group. ${ }^{1} A P-H P$, Hôpital Cochin; Centre Référence Maladies Rares, Service de Médecine interne, Paris, France; ${ }^{2}$ University of Padova, Rheumatology Unit, Department of Medicine-DIMED, Padova, Italy; ${ }^{3}$ Centre Hospitalier Universitaire de Bordeaux, Service De Médecine Interne et Maladies Infectieuses, Bordeaux, France; ${ }^{4}$ AP-HP, Hôpital Saint-Antoine, Service de Médecine interne, Paris, France; ${ }^{5} \mathrm{CH}$ Annecy Genevois, Service Infectiologie et médecine interne, Annecy, France; ${ }^{6} \mathrm{CHU}$ de Reims-Robert Debré, Service de Médecine interne, Reims, France $;{ }^{7} \mathrm{CHRU}$ de Tours - Hôpital Bretonneau, Service de Médecine interne, Tours, France; ${ }^{8} \mathrm{CHU}$ Grenoble, Service de Médecine interne, Grenoble, France; ${ }^{9}$ AP-HP, Hôpital Cochin, Service de Rhumatologie B, Paris, France; ${ }^{10}$ Epidemiology and Statistics Research Center/CRESS, INSERM, INRA F-75004, Equipe Épope, Paris, France

Background: Active Systemic Lupus Erythematosus (SLE) during pregnancy is associated with poor obstetrical outcome but it is still not clear if remission, lupus low disease activity state (LLDAS) is the best target to achieve at conception. Besides, the effect of damage on pregnancy outcome has not been studied.

Objectives: Our aim was to determine the $1^{\text {st }}$ trimester risk factors for adverse pregnancy outcome (APO).

Methods: Inclusion criteria were: 1 ) women $\geq 18$ years enrolled in the prospective GR2 study; 2) with SLE (SLICC criteria); 3) and an ongoing singleton pregnancy at 12 weeks (only 1 pregnancy per patient). We used the following definitions: DORIS $^{1}$, DORIA $^{2}$, clinical SLEDAI-2K=0, LLDAS $^{3}$ (for SLE activity), $\mathrm{SFI}^{4}$ (for flares), and SLICC-damage index ${ }^{5}$ (for damage). APO included: foetal death, neonatal death, placental insufficiency with premature delivery $<37$ weeks, and small for gestational age (SGA: $\leq 3^{\text {rd }}$ percentile).

Results: 238 patients were included. 234 (98.3\%) women were on hydroxychloroquine (HCQ) and $206(86.5 \%)$ had a clinical SLEDAI-2K=0. Regarding pregnancy outcome, 230 (96.6\%) patients had a live birth (mean term 37.7 weeks). Thirty-four (14.3\%) patients developed at least 1 APO: placental insufficiency

Table 1.

Univariate analysis for APO

\begin{tabular}{|c|c|c|c|c|}
\hline Maternal features & Total $(\mathrm{N}=238)$ & APO $(\mathrm{N}=34)$ & Non-APO ( $\mathrm{N}=204)$ & $P$ value \\
\hline Age, mean (SD) & $31.6(4.5)$ & $30.7(4.8)$ & $31.7(4.4)$ & 0.22 \\
\hline Secondary APS & $34(14.3)$ & $10(29.4)$ & $24(11.8)$ & 0.01 \\
\hline Previous renal phenotype & $67(28.2)$ & $13(38.2)$ & $54(26.5)$ & 0.16 \\
\hline At least 1 flare during pregnancy & $37(15.5)$ & $6(17.4)$ & $31(15.2)$ & 0.80 \\
\hline Positive anti-DNA ( $\mathrm{N}=222)$ & $104(46.8)$ & $21(67.7)$ & $83(43.5)$ & 0.01 \\
\hline Hypocomplementemia $(\mathrm{N}=216)$ & $57(26.4)$ & $13(40.6)$ & $44(23.9)$ & 0.05 \\
\hline LAC $(\mathrm{N}=232)$ & $41(17.7)$ & $15(44.1)$ & $26(13.1)$ & $<0.001$ \\
\hline Triple aPL (N=232) & $17(7.3)$ & $5(14.7)$ & $12(6.1)$ & 0.08 \\
\hline $24 \mathrm{~h}$-proteinuria $>0.5 \mathrm{~g} /$ day & $9(3.8)$ & $3(8.8)$ & $6(2.9)$ & 0.12 \\
\hline \multicolumn{5}{|l|}{ Activity/Damage } \\
\hline SLEDAI-2K, median (IQR) $(\mathrm{N}=212)$ & $2(0-3)$ & $2(2-4)$ & $2(0-2)$ & 0.01 \\
\hline SLICC-DI, median (IQR) $(\mathrm{N}=236)$ & $0(0-0)$ & $0(0-0)$ & $0(0-0)$ & 0.007 \\
\hline PGA, median (IQR)(N=235) & $0.1(0-0.2)$ & $0.1(0-0.41)$ & $0.1(0-0.2)$ & 0.06 \\
\hline DORIA remission* & $154(64.7)$ & $17(50.0)$ & 137(67.2) & 0.05 \\
\hline DORIS remission** & $147(61.8)$ & $17(50.0)$ & $130(63.4)$ & 0.13 \\
\hline LLDAS (N=219) & $157(71.7)$ & $19(57.6)$ & 138(74.2) & 0.05 \\
\hline Clinical SLEDAI-2K=0 & $206(86.5)$ & $28(82.4)$ & $178(87.3)$ & 0.44 \\
\hline \multicolumn{5}{|l|}{ Treatment } \\
\hline Prednisone (PDN) & $119(50.0)$ & $23(67.7)$ & $96(47.1)$ & 0.03 \\
\hline PDN (mg/day) median (IQR) & $7(5-10)$ & $0(0-6)$ & $5(0-10)$ & 0.007 \\
\hline Immunosuppressants & $57(24.0)$ & 13(38.2) & $44(21.6)$ & 0.04 \\
\hline Hydroxychloroquine & $234(98.3)$ & $34(100.0)$ & $200(98.0)$ & 1.00 \\
\hline Low dose aspirin & $165(69.3)$ & $29(85.3)$ & $136(66.7)$ & 0.03 \\
\hline Low molecular weight heparin & $61(25.6)$ & $15(44.1)$ & $46(22.6)$ & 0.01 \\
\hline
\end{tabular}

Legend: APS: antiphospholipid syndrome; aPL: antiphospholipid; PGA: Physician global assessment. *: DORIA definition of remission = clinical SLEDAl $=0$ and prednisone $\leq 5 \mathrm{mg} /$ day;

${ }^{* *}$ : DORIS definition of remission = clinical SLEDAI $=0$, prednisone $\leq 5 \mathrm{mg} / \mathrm{day}$, and $\mathrm{PGA}<0.5$. $(n=22)$, foetal death $(n=7)$, neonatal death $(n=1)$, and SGA $(n=5)$. Two different regression logistic models were assessed, one for DORIA and one for LLDAS. We found that only SLICC-Damage index and lupus anticoagulant (LAC) were associated with APO $(\mathrm{p}=0.02$, OR $1.8,95 \% \mathrm{Cl}: 1.1-2.9 ; \mathrm{p}=0.001$, OR $4.2,95 \% \mathrm{Cl}$ : 1.8-9.7 respectively for DORIA model; $p=0.03$, OR 1.7, 95\% Cl:1.1-2.8; $p=0.002$ OR 3.7, 95\% Cl: 1.6-8.7 respectively for LLDAS model).

Conclusion: We confirmed that LAC predicts APO. We found for the first time that chronic damage at $1^{\text {st }}$ trimester also predicted APO. No effect of remission/LLDAS was observed in this cohort of patients on HCQ with a stable and well-controlled SLE.

\section{REFERENCES:}

[1] van Vollenhoven R, et al. ARD 2017.

[2] Zen M, et al. ARD. 2015.

[3] Franklyn, K. et al. ARD 2016.

[4] Petri M, et al. NEJM 2005.

[5] Gladman DD, et al. Arthritis Rheum, 1997.

Disclosure of Interests: None declared.

DOI: 10.1136/annrheumdis-2021-eular.3876

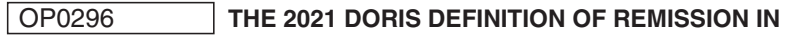 SLE - FINAL RECOMMENDATIONS FROM AN INTERNATIONAL TASK FORCE}

R. van Vollenhoven ${ }^{1,2}$, G. Bertsias ${ }^{3}$, A. Doria ${ }^{4}$, D. Isenberg ${ }^{5}$, E. F. Morand ${ }^{6}$, M. A. Petri ${ }^{7}$, B. Pons-Estel ${ }^{8}$, A. Rahman ${ }^{5}$, M. Ugarte-Gil ${ }^{9}$, A. Voskuyl10,11,

L. Arnaud ${ }^{12}$, I. N. Bruce ${ }^{13}$, R. Cervera ${ }^{14}$, N. Costedoat-Chalumeau ${ }^{15}$,

C. Gordon ${ }^{16}$, F. Houssiau ${ }^{17}$, M. Mosca ${ }^{18}$, M. Schneider ${ }^{19}$, M. Ward ${ }^{20}$,

C. Aranow ${ }^{21}$ on behalf of The DORIS Task Force. ${ }^{1}$ Amsterdam UMC,

Rheumatology and Clinical Immunology, Amsterdam, Netherlands;

${ }^{2}$ Amsterdam Rheumatology Center, Rheumatology, Amsterdam,

Netherlands; ${ }^{3}$ University of Crete, Rheumatology, Heraklion, Greece;

${ }^{4}$ University of Padova, Medicine, Padova, Italy; ${ }^{5}$ University College London, Rheumatology, London, United Kingdom; ${ }^{6}$ Monash University, School of Clinical Sciences, Melbourne, Australia; ${ }^{7} J o h n s$ Hopkins University, Rheumatology, Baltimore, United States of America; ${ }^{8}$ Grupo Oroño - Centro Regional de Enfermedades Autoinmunes y Reumáticas (GO-CREAR)., n/a, Rosario, Argentina; ${ }^{9}$ Universidad Cientifica del Sur, Medicine, Lima, Peru; ${ }^{1}$ Amsterdam UMC, Rheumatology and Clinical Immunology, Amsterdam, Netherlands; ${ }^{2}$ Amsterdam Rheumatology Center, Rheumatology, Amsterdam, Netherlands; ${ }^{12}$ University Hospitals of Strasbourg, Rheumatology, Strasbourg, France; ${ }^{13}$ University of Manchester, Division of Musculoskeletal \& Dermatological Sciences, Manchester, United Kingdom; ${ }^{14} \mathrm{Hospital}$ Clinic, Autoimmune Diseases, Barcelona, Spain; ${ }^{15}$ AP-HP, Cochin Hospital, Internal Medicine, Paris, France; ${ }^{16}$ University of Birmnigham, Rheumatology, Birmingham, United Kingdom; ${ }^{17}$ Cliniques universitaires Saint-Luc, Rheumatology, Brussels, Belgium; ${ }^{18}$ University of Pisa, Rheumatology, Pisa, Italy; ${ }^{19}$ Heinrich-Heine University, Rheumatology, Dusseldorf, Germany;

${ }^{20}$ NIAMS/NIH, Intramural Research Program, Bethesda, United States of America; ${ }^{21}$ Feinstein Institute for Medical Research, n/a, Manhasset, United States of America

Background: Remission is the stated goal for both patient and care-giver (1) but consensus on a definition of remission has been lacking. Previously, an international task force consisting of patient representatives and medical specialists published a frame-work for such a definition (2), but without making a final recommendation.

Objectives: To achieve consensus around a definition of remission in SLE (DORIS).

Methods: The DORIS task force met annually from 2015 to 2020 and consisted of patient representatives and specialists in rheumatology, nephrology, dermatology, and clinical immunology. Systemic literature reviews of severa key topics were done and specific research questions were examined in suitably chosen datasets. The findings were discussed, reformulated as recommendations, and voted upon. Level of evidence (LoE), strength of recommendation (SoR), and agreement were determined in standard fashion. The final recommendation for the DORIS definition of remission was established by electronic vote after finalization of the minutes of the most recent task force meeting.

Results: Based on data from the literature and from several SLE-specific data sets, five key recommendations were endorsed (Table 1) that should be seen as additions to those published previously (2). Literature reviews identified strong support for the face-, content-, construct- and criterion validity of the definition based on the clinical SLEDAI (not including anti-DNA and complement) equa to zero plus low physician global assessment and allowing stable medical treatment. Thus, the DORIS Task Force recommended a single definition of remission in SLE, based on clinical SLEDAI $=0$, evaluator's global assessment $<0.5(0-3)$, 